\title{
Study on Calculation Method of Economic Velocity of Gas Phase Ethane Conveying Pipeline
}

\author{
Yuzhu Wang ${ }^{\mathrm{a}}$, Xiaobo Mou ${ }^{\mathrm{a}}$, Yuanrui Zhang ${ }^{\mathrm{b}}$, Lande Cui ${ }^{\mathrm{a}}$, Wenlong Jia ${ }^{\mathrm{b}}$ \\ ${ }^{a}$ Oil and Gas Engineering Research Institute, Petrochina Tarim Oilfield Company, Korla, \\ Xinjiang,841000, China \\ ${ }^{b}$ School of Petroleum and Natural Gas Engineering, Southwest Petroleum University, Chengdu, \\ Sichuan,610500, China.
}

Received: 27 September 2019; Accepted: 15 October 2019; Published: 08 November 2019

\begin{abstract}
Ethane is different from natural gas, crude oil, etc., its critical temperature is low, and it is easy to change phase with the change of ambient temperature. Therefore, it is necessary to increase the constraint condition to ensure that ethane is in the gas phase. Therefore, based on the optimization principle, the calculation model of the economical flow rate of the ethane transport pipeline including the ethane phase, the pipeline stability, and the compressor pressure ratio constraint is established with the lowest annual cost of the gas phase ethane pipeline. Solve the problem with the lowest cost. The gas-phase ethane transmission pipeline proposed in Tarim Oilfield is analyzed as an example. The results show that the economical flow rate of the pipeline under different working conditions ranges from 3 to $7 \mathrm{~m} / \mathrm{s}$, which guides the selection of the diameter of the Tarimethane pipeline. The economic design, safe operation and management of the ethane pipeline provide the basis.
\end{abstract}

Index Terms: Gas-Phase Ethane; Pipeline; Economic Flow Rate; Mathematical Model; Calculation

(C) 2019 Published by MECS Publisher. Selection and/or peer review under responsibility of the Research Association of Mode rn Education and Computer Science

* Corresponding author.

E-mail address: zhangyuanrui05@sina.com 


\section{Introduction}

In the petrochemical industry, ethylene is an important raw material, and there are many derivatives, including polyethylene, ethylene glycol, styrene, etc., and ethane is the most economical material for ethylene [1-3]. In natural gas production, the main purpose of ethane removal is to make the natural gas's hydrocarbon dew point meet the external demand, rather than the large-scale use of ethane. However, in recent years, in the development of the Tarim Oilfield, a large number of high-temperature and high-pressure condensate gas fields such as Dina and Yaha have been discovered, and the volume of ethane in the produced condensate gas is higher than $5 \%$, which is a large-scale removal of $\mathrm{B}$. The use of alkane for scale application provides good resource conditions. For long-distance onshore transport of large amounts of ethane, pipeline transport is the most convenient and economical means of transport.

A large number of ethane pipeline systems that can match natural and crude oil pipelines have been established abroad, mainly in Australia, North America, and the Middle East. For example, North American EPP (Enterprise Products Partners)'s "Aegis" ethane pipeline system[4], Alberta ethane collection system, Australia APA's Momba-Sydney liquid phase ethane pipeline[5], Saudi Aramco's gas-rich ethane transfer pipeline[6], etc., these pipelines are more than hundreds of kilometers in length. In the long-distance ethane pipeline that has been built, there are both liquid phase transportation and gas-phase transportation. In contrast, China's long-distance ethane pipeline is still in its infancy. Compared with the liquid phase transportation mode, the gas phase transportation method is simpler, so this paper mainly studies the gas phase pipeline.

The economical flow rate is a fundamental parameter that is important in the design and operation of a gas phase ethane pipeline. At present, the design standards for gas pipelines at home and abroad are mainly for the establishment of natural gas, and the design standards and specifications for gas-phase ethane pipelines have not yet been formed[7-9]. The research on the economical flow rate of gas-phase ethane pipelines is rarely reported. Although the design of gas-phase ethane pipeline can refer to the relevant standards of natural gas transmission pipeline, the physical parameters such as bubble point, dew point, viscosity and density of ethane are quite different from those of natural gas, liquefied petroleum gas and crude oil, and the related pipelines. The energy consumption of transportation must be different. Especially for commercial ethane with a purity of more than $95 \%$, the critical pressure and critical temperature are about $4.9 \mathrm{MPa}$ and $32{ }^{\circ} \mathrm{C}$, respectively. It is easy to change phase at normal temperature and cause flow safety problems such as pipeline effusion and slug flow. Therefore, in determining the economical flow rate of the gas phase ethane delivery pipeline, it is necessary to consider not only the economic factors such as operating energy consumption but also the gas-liquid phase change factor of ethane. To this end, it is necessary to combine the physical property parameters of ethane and the transportation process to clarify the economical flow rate of the long-distance pipeline of the gas-phase ethane and provide guidance for the safe, economical and efficient design and operation management of the gas-phase ethane pipeline.

\section{Gas-phase ethane pipeline transportation process}

In natural gas processing plants, ethane is generally derived from natural gas de-light hydrocarbon plants, mostly in a gas phase with high temperature and low pressure. Before entering the pipeline, the gas phase ethane needs to be pressurized to meet the pipeline requirements. During the transportation process, ethane is kept in the gas phase and continuously conveyed by the compressor pressurization. The pipe delivery process is shown in Fig.1. The critical pressure of ethane is $4.88 \mathrm{MPa}$ and the critical temperature is $32.25^{\circ} \mathrm{C}$. It should be controlled at 2 2.5 MPa during the transportation process and the temperature is controlled at $0 \sim 25{ }^{\circ} \mathrm{C}$. Otherwise, it is easy to cause the gas phase ethane to liquefy, enter the liquid phase or supercritical state, and form multiphase flow to affect the normal operation of the pipeline and equipment. 


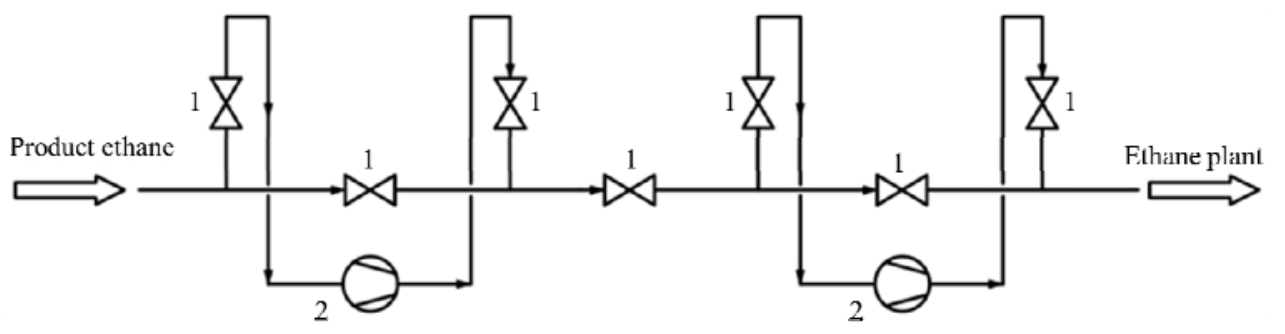

Fig. 1. Gas-phase ethane pipeline transportation process.1- valve; 2- compressor.

\section{Economic flow rate calculation model}

The investment cost of the gas phase ethane long-distance pipeline construction is high, the pipeline operation time is long, and the operation and maintenance cost is large. Whether the economic operation flow rate of the ethane pipeline can be determined is directly related to whether the pipeline construction is economically feasible. To determine the economic flow rate of the pipeline, the principle to be followed is that the total investment cost of the pipeline is the lowest, so an optimization model needs to be established to obtain the economic flow rate.

Combined with the characteristics of long-distance pipeline construction and operation, the total investment cost of the long-distance gas pipeline of ethane can be obtained, including construction cost and operation cost. The construction cost includes pipeline construction, land cost, gas station construction, etc. The operating cost includes the compressor unit. The electricity cost of the valve room is equal[10-13]. According to the above characteristics, the annual equivalent cost of the gas phase ethane long-distance pipeline is used as the objective function, and the mathematical expression is as shown in the formula:

$$
S=\left(S_{\mathrm{A}}+S_{\mathrm{B}}\right) \cdot E+S_{\mathrm{C}}+S_{\mathrm{D}}
$$

\subsection{Annual equivalent Pipeline Investment (SA)}

The construction cost of the ethane pipeline is mainly comprised of construction cost independent of pipeline diameter, cost of anti-corrosion coating and pipe material, etc [11,12]. The specific formula is as follows:

$$
S_{\mathrm{A}}=\left(a_{0}+a_{1} D+a_{2} \delta(D-\delta)+a_{3} D \pi+a_{4}(D-2 \delta)\right) \cdot L
$$

\subsection{Annual Equivalent Station Investment (SB)}

For the long-distance gaseous ethane pipeline, it is also considered that intermediate pressurization and compressor station. Station construction investment can be divided into two parts, one is station construction costs, including office buildings, fire facilities and so on, and the other is the compressor as well as other equipment investment. The formula for investment in station construction can be expressed as follows:

$$
S_{\mathrm{B}}=m \cdot\left(b_{0}+b_{1} N\right)
$$

Note: In practice, the arguments of $b_{0}$ and $b_{1}$ varies in different compressor station due to their various versions and specifications as well as different types of connection. Therefore, to unify all arguments and 
simplify the model, all compressor stations considered the same standard one.

For the booster station, according to the gas ethane piping in the low-pressure transmission, the electrically driven centrifugal compressor can be chosen, its power can be decided as follows:

$$
N=\frac{4 k}{\eta(k-1)} \cdot Q Z T\left(\varepsilon^{\frac{k-1}{k}}-1\right) \cdot 10^{-6}
$$

\subsection{Operation and maintenance costs (SC)}

It is necessary to invest to maintain the day-to-day operation of pipelines and stations after they are completed and put into operation. Therefore, the operation and maintenance costs are calculated from the end of the construction. According to the economic evaluation of Pipeline Engineering, the annual operation cost of pipeline and stations can be calculated as follows:

$$
S_{\mathrm{C}}=C_{0} S_{\mathrm{A}}+C_{1} S_{\mathrm{B}}
$$

\subsection{Annual Energy Consumption Cost(SD)}

The annual energy consumption of the station mainly includes the electricity cost of the compressor, and the remaining electricity cost can be calculated by the additional energy consumption factor. The formula is:

$$
S_{\mathrm{D}}=m \lambda Y_{\mathrm{e}} N H_{\mathrm{d}} \times 24
$$

\subsection{Annual Equivalent Factor(E)}

Given that the time cost of the pipeline and the station in combination with annual energy consumption cost, the annual equivalent factor is adopted to unify the cost formula as follows:

$$
E=\frac{i_{0}\left(1+i_{0}\right)^{y}}{\left(1+i_{0}\right)^{y}-1}
$$

\section{Piping constraint conditions}

A long-distance gas transmission pipeline, generally, is a complex hydraulic system. In the process design, the corresponding constraints must be met to achieve the goal of safe operation after construction. The pipeline design is a matter of optimization, in which various constraints should be factored into, including phase state constraints, pipeline strength constraints, hydraulic constraints, pipe specifications, and compressor spacing, etc.

(1). Phase Constraints

Phase state constraint means that the pressure at any temperature in the gas phase is less than the related dew-point pressure at that temperature. The constraints are as follows:

$$
\left(P_{\mathrm{x}}, T_{\mathrm{x}}\right)<\left(P_{\text {dew }}, T_{\text {dew }}\right)
$$

(2). Pipeline Strength Constraint 
Because the maximum pressure is located at the outlet of the compressor station, the constraint pressure in the constraint condition is chosen as the outlet pressure of the compressor station. The Piping strength constraint formula is as follows:

$$
\delta \geq \frac{P D}{2 \sigma_{s} \varphi F t}
$$

(3). Pipe and pipe specification constraint

The pipes used in gas pipelines are manufactured according to a certain standard series, so the pipe diameter and wall thickness are not continuous variables, but discrete values that are compatible with the specifications, so the pipeline The pipe diameter and wall thickness should be selected from the relevant standards for the specified pipe diameter and wall thickness.

$$
D \subset \bar{D}, \quad \delta \subset \bar{\delta}
$$

(4). Pipeline stability constraint

The instability of the cross-section of the long-distance pipeline should be considered when it is buried. The stability constraint is as follows:

$$
80 \leq \frac{D}{\delta}=E_{\mathrm{p}} \leq 120
$$

(5). Power constraint of compressors

To make sure that the compressor work in a high-efficiency area working area and improve the working efficiency, the range of the compressor's pressure ratio has to be determined before the compressor station's pressure ratio is determined. Therefore, the compressor station pressure ratio should be in a certain range, as shown in the following formula:

$$
\varepsilon_{\min } \leq \varepsilon \leq \varepsilon_{\max }
$$

(6). Hydraulic condition constraint

When the piped gas flows in the pipe, it will be subjected to frictional resistance, resulting in a change in the gas transporting capacity, so the gas flowing in the pipe needs to meet the corresponding hydraulic conditions.

The overall constraint model is:

$$
\begin{aligned}
& \min S=\left(S_{\mathrm{A}}+S_{\mathrm{B}}\right) \cdot E+S_{\mathrm{C}}+S_{\mathrm{D}} \\
& \text { s.t. }\left\{\begin{array}{l}
P_{x}<P_{d e w} \\
\delta \geq \frac{P D}{2 \sigma_{s} \varphi F t} \\
D \subset \bar{D}, \quad \delta \subset \bar{\delta} \\
80 \leq \frac{D}{\delta}=E_{\mathrm{p}} \leq 120 \\
\varepsilon_{\min } \leq \varepsilon \leq \varepsilon_{\max }
\end{array}\right.
\end{aligned}
$$


Solve the mathematical model according to the following diagram.

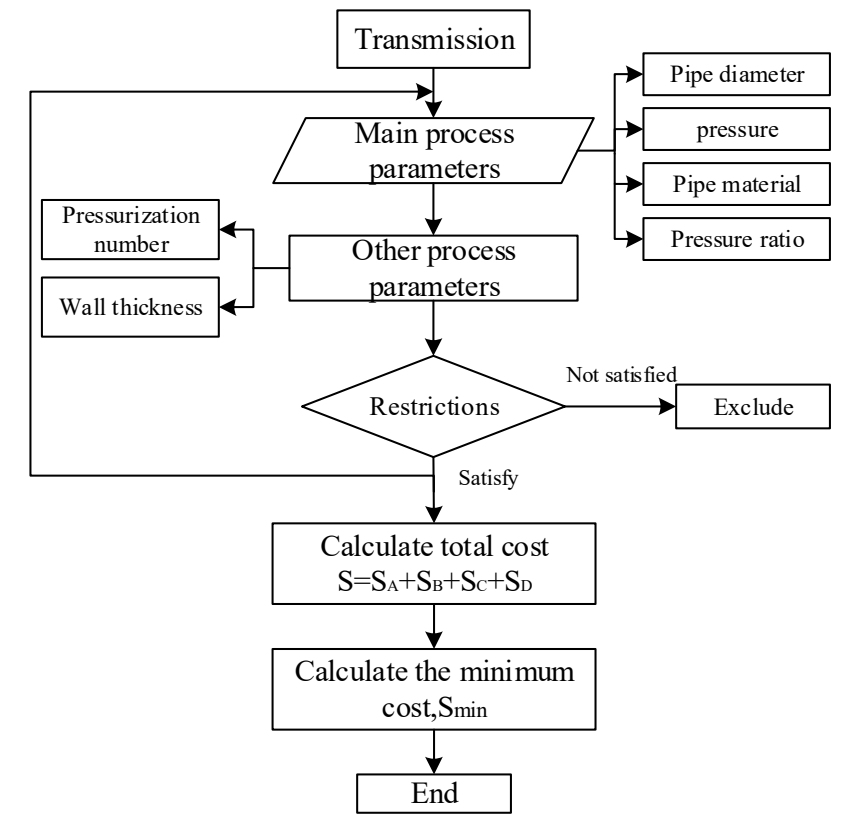

Fig. 2. Calculation method flow chart

\section{Case Studies}

\subsection{Case 1}

Case analysis of the Lunnan-to-Korla gas-phase ethane pipeline proposed by Tarim Oilfield. The length of the pipeline is $125 \mathrm{~km}$, the terrain is flat and the total output is $2286 \mathrm{t} / \mathrm{d}$. The outlet pressure is $2.1 \mathrm{MPa}$ and the minimum inlet pressure is $1.2 \mathrm{MPa}$, meanwhile, the outlet temperature is $29^{\circ} \mathrm{C}$ above the ambient temperature which is $20{ }^{\circ} \mathrm{C}$ in summer while $3{ }^{\circ} \mathrm{C}$ in winter. The detail ethane components are shown in Table 1 below. Referring to the economic evaluation arguments of domestic oil and gas company projects and relevant documents or literature ${ }^{[14,15]}$, the values of each economic parameter are determined, as shown in Table 2 below.

Table 1. Composition of Ethane Products

\begin{tabular}{ll}
\hline Composition & proportion $(\mathrm{mol} \%)$ \\
\hline $\mathrm{C} 1$ & 1.426 \\
$\mathrm{C} 2$ & 96.262 \\
$\mathrm{C} 3$ & 2.282 \\
\hline
\end{tabular}


Table 2. Economic Arguments

\begin{tabular}{ll}
\hline Economic arguments & Parameter value \\
\hline Cost factor independent to the diameter & 52.0639 \\
Construct cost factor of pipeline & 0.8244683 \\
The cost factor of pipeline materials & 0.62 \\
The cost factor of external anti-corrosion coating & 8 \\
The cost factor of internal anticorrosion coating & 4 \\
Cost factor independent to station buildings & 588.9 \\
Cost factor related to power equipment & 1.36 \\
annual operating maintenance factor of pipeline & 0.02 \\
annual operating maintenance factor of station & 0.02 \\
\hline
\end{tabular}

After all, after the optimization calculation, the optimal solution is shown in the following tablet, the economic flow rate of the pipeline is $4.4 \mathrm{~m} / \mathrm{s}$. The starting pressure is $2.1 \mathrm{MPa}$ and the starting temperature is $29{ }^{\circ} \mathrm{C}$, which is namely the outlet pressure and temperature of ethane plant, hence no extra compressor or heat exchanger is needed. The profile of pressure and temperature in the whole line is shown in fig. 3 below, which meets the requirement for gaseous ethane transmission.

Table 3. Optimal delivery scheme

\begin{tabular}{|c|c|c|c|c|}
\hline Pipe diameter $/ \mathrm{mm}$ & flow rate $/(\mathrm{m} / \mathrm{s})$ & pump station & inner coating & outer coating \\
\hline $559 \times 6.4$ & 4.4 & no & no & yes \\
\hline
\end{tabular}

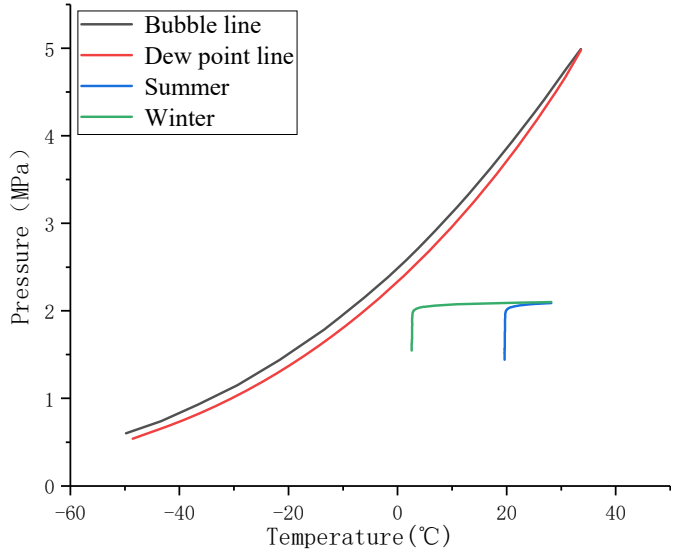

Fig. 3. profile of pressure and temperature

Through the analysis of the economic model, it can be concluded that pipeline throughput and distance are the two most important factors affecting the model. Therefore, to decide the optimal method, specifically, optimal economic flow rate, it must be done that changing the two parameters and forming various schemes to compare economic estimate. As shown in Fig.4 and Fig.5, the economical flow rate of ethane pipeline range is $3 \sim 7 \mathrm{~m} / \mathrm{s}$. 


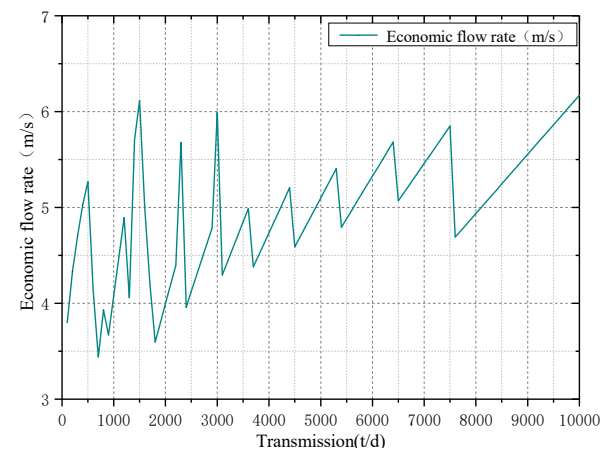

Fig. 4. economic flow rate with different throughput

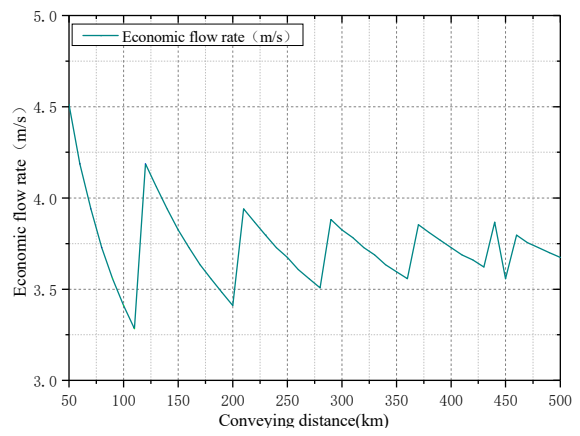

Fig. 5. economic flow rate with different transmission distance

\subsection{Case 2}

The length of the pipeline is $4300 \mathrm{~km}$, and the elevation along the pipeline is as shown in fig.6. The total output is $4000 \mathrm{t} / \mathrm{d}$. The pressure of the ethane plant is $2.0 \mathrm{MPa}$, the minimum pit pressure is $1.2 \mathrm{MPa}$, the starting temperature is $29.0^{\circ} \mathrm{C}$, and the ambient temperature is 3 to $20.0^{\circ} \mathrm{C}$. The composition of the transport medium is shown in Table 1, and the values of the economic parameters are shown in Table 2.

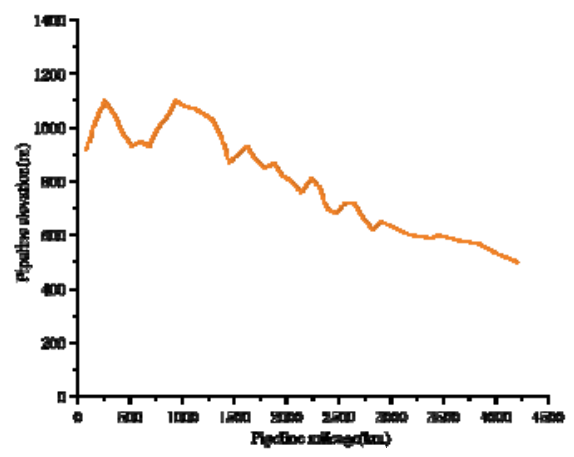

Fig. 6. elevation distribution along the pipeline 


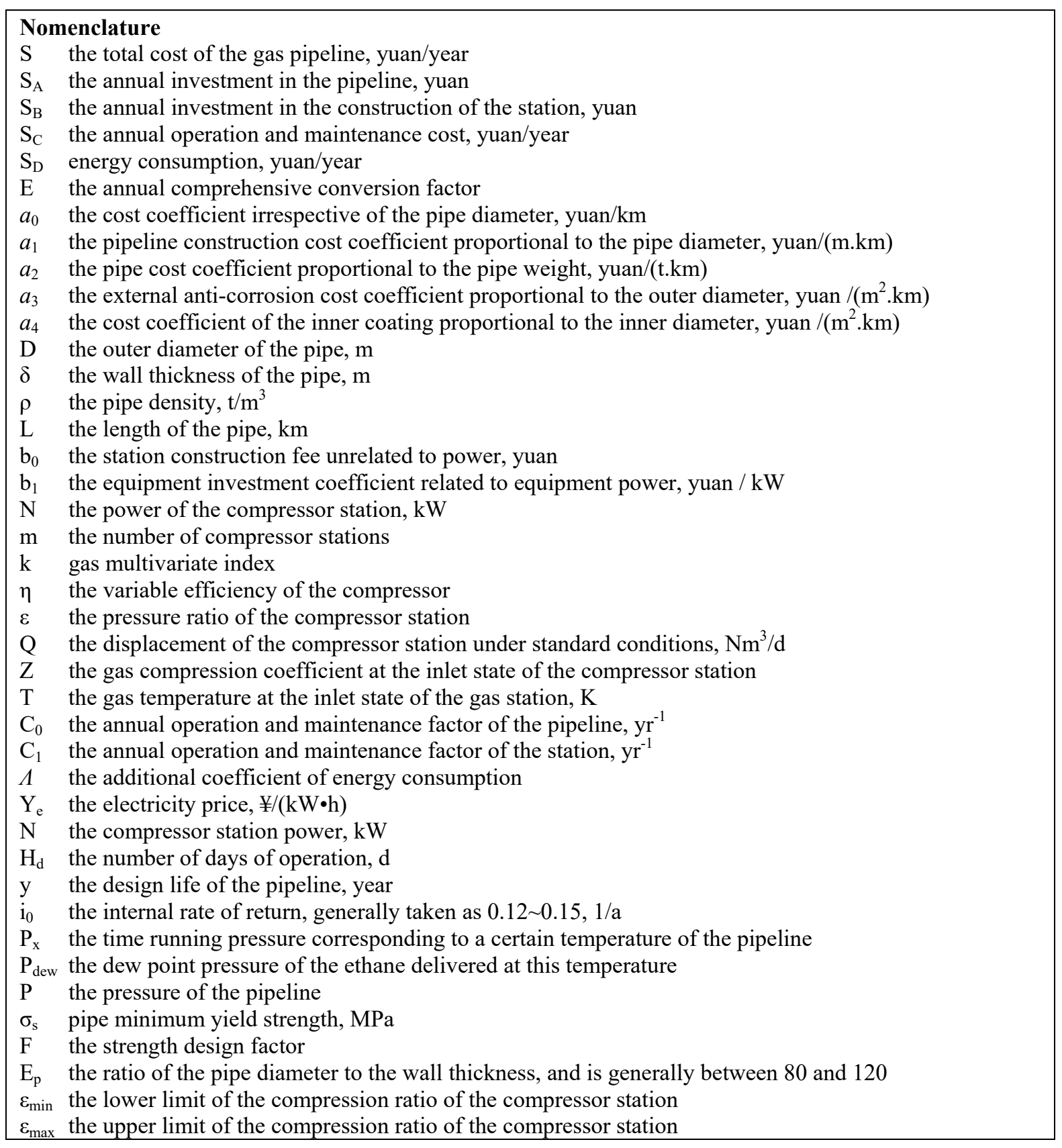


By optimizing the calculation, the optimal solution is shown in the table4 below. The economical flow rate of pipeline transportation is $4.7 \mathrm{~m} / \mathrm{s}$. Change the output, form a variety of programs, and get the economic flow rate under different working conditions, as shown in the figure. As can be seen from the fig.7, the economic flow rate in this example is between 3.5 and $5.5 \mathrm{~m} / \mathrm{s}$.

Table 4. Optimal delivery scheme

\begin{tabular}{lllll}
\hline Pipe diameter $/ \mathrm{mm}$ & flow rate $/(\mathrm{m} / \mathrm{s})$ & pump station & inner coating & outer coating \\
\hline $660 \times 9$ & 4.7 & 22 & no & yes \\
\hline
\end{tabular}

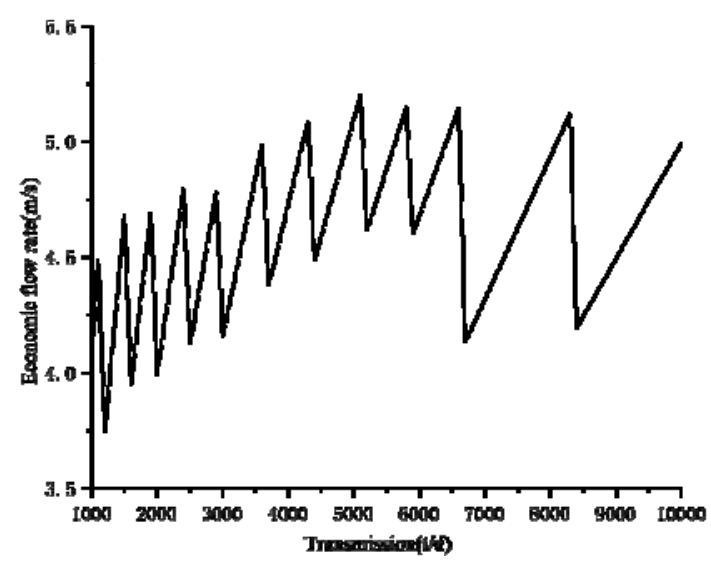

Fig. 7. economic flow rate under different inputs

\subsection{Case 3}

The length of the pipeline is $1000 \mathrm{~km}$, and the terrain is flat and the total output is $4000 \mathrm{t} / \mathrm{d}$. The pressure of the ethane plant is $1.8 \mathrm{MPa}$, the minimum pit pressure is $1.3 \mathrm{MPa}$, the starting temperature is $33^{\circ} \mathrm{C}$, and the ambient temperature is $3-15^{\circ} \mathrm{C}$. The composition of the transport medium is shown in Table 1 , and the values of the economic parameters are shown in Table 2.

Through optimization calculation, the economical flow rate of pipeline transportation under the optimal scheme is $4.6 \mathrm{~m} / \mathrm{s}$. Change the output, form a variety of programs, and get the economic flow rate under different working conditions, as shown in the fig.8. The figure shows. The economic flow rate under this example is between 3.0 and $5.0 \mathrm{~m} / \mathrm{s}$.

Table 5. Optimal delivery scheme

\begin{tabular}{lllll}
\hline Pipe diameter $/ \mathrm{mm}$ & flow rate $/(\mathrm{m} / \mathrm{s})$ & pump station & inner coating & outer coating \\
\hline $660 \times 9$ & 4.6 & 6 & no & yes \\
\hline
\end{tabular}




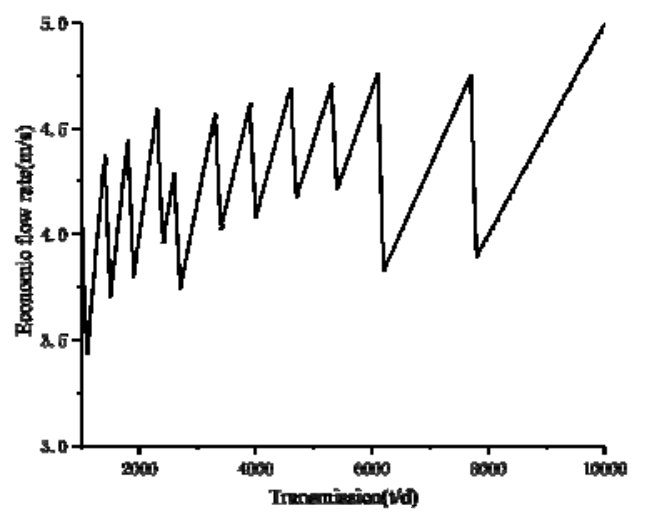

Fig. 8. economic flow rate under different inputs

In summary, the economical flow rate of the gas phase ethane pipeline is $3 \sim 7 \mathrm{~m} / \mathrm{s}$. In contrast, the economic flow rate of conventional natural gas pipelines is $7 \sim 15 \mathrm{~m} / \mathrm{s}$, the flow rate of crude oil is $1-2 \mathrm{~m} / \mathrm{s}$, and the economic flow rate of ethane pipelines is between conventional natural gas and crude oil pipelines. between. This is mainly because commercial ethane and density are between natural gas and crude oil. The friction loss generated under the same output is inevitably between natural gas and crude oil, and the corresponding economic flow rate will not exceed the crude oil and natural gas pipeline. The scope. The economical flow rate of the established Saudi Aramco NGP gas-phase ethane pipeline is about $4.5 \sim 9.14 \mathrm{~m} / \mathrm{s}$, which is consistent with the calculation results in this paper.

Fig. 4 and Fig. 5 also show that the economic flow rate of the ethane transfer line fluctuates within a range, which is formed by the influence of the standard pipe diameter of the pipe. For example, in Fig.4, the conditions such as the transport distance of $125 \mathrm{~km}$ are the same as in the example, and only the pipeline output is changed. When the output is $5500-6500 \mathrm{t} / \mathrm{d}$, the outer diameter of the pipe is $610 \mathrm{~mm}$, which is the optimal pipe diameter. At this time, the economic flow rate increases with the increase of the output. When the output is increased to $6500 \mathrm{t} / \mathrm{d}$, the optimal pipe outer diameter is increased from $610 \mathrm{~mm}$ to $660 \mathrm{~mm}$, the pipe flow rate is decreased, and then increases with the increase in the number. In Fig.5, the conditions such as the pipe volume of $2286 \mathrm{t} / \mathrm{d}$ are the same as in the example, and only the conveying distance is changed. When the conveying distance is $210-270 \mathrm{~km}$, as the conveying distance increases, the flow rate can be reduced by increasing the pipe diameter to reduce the friction loss. At this time, the cost of increasing the pipe diameter is less than the construction and operation cost of the gas station, so the pipe flow rate is reduced. However, when the conveying distance reaches $280 \mathrm{~km}$, the cost of increasing the pipe diameter is greater than the construction and operation cost of the compressor station. At this time, the pressure station can be increased, the pipe diameter can be reduced, and the pipe flow rate can be increased accordingly.

\section{Conclusion}

(1) Considering the ethane physical property and gas-phase pipeline transportation process, based on the optimization principle, the lowest annual cost of the gas phase ethane delivery pipeline is established as the objective function, including the ethane phase, pipeline stability, pipeline strength, and compressor pressure ratio constraint. The economical flow rate calculation model of the ethane transfer pipeline.

(2) The model was applied to the proposed Tarim gas-phase ethane pipeline, which guided the selection of the flow rate of the Tarimethane pipeline. And by changing the conveying conditions, the economical flow 
rate under various schemes is $3 \sim 7 \mathrm{~m} / \mathrm{s}$, which is consistent with the flow rate of pipelines built at home and abroad and has a guiding role for the construction of gas-phase ethane pipelines.

(3) At present, the theoretical design, and calculation of the gas phase ethane pipeline transportation process are carried out, and there is not enough practical argumentation. In the future, the actual economic situation and different pipeline materials in different regions can be combined to further refine the model and establish a more complete economic flow calculation model for the gas phase ethane pipeline.

\section{References}

[1] Bohong Wang, Haoran Zhang, Meng Yuan, etc. Sustainable crude oil transportation: design optimization for pipelines considering thermal and hydraulic energy consumption. Chemical Engineering Research and Design 2019;151:23-29.

[2] Design optimization of oilfield subsea infrastructures with manifold placement and pipeline layout. Computers \& Chemical Engineering 2018;108:163-178.

[3] Multilevel modeling and optimization of large-scale pipeline systems operation. Energy 2019;184:151-164.

[4] Lind G, Bachman S. Lessons, Issues, and Perils Experienced During the Conceptual Design and Predictive Modeling of an Ethane Pipeline. PSIG Annual Meeting. Pipeline Simulation Interest Group, 2012.

[5] Coles, R.B.. (1996). ICI Australia Moomba-Sydney 1375 km ethane pipeline: pipeline to the world. 84 . 34-37.

[6] Raed Zarei , Carlos Alvarez. Transport of Ethane-Rich Gases Using an Extensive Gas Pipeline System[C]. Society of Petroleum Engineers.

[7] H. Zhang, Y. Liang, J. Ma, Y. Shen, X. Yan, M. Yuan. An improved PSO method for optimal design of subsea oil pipelines. Ocean Engineering 2017;141:154-163

[8] C. Zeng, C. Wu, L. Zuo, B. Zhang, X. Hu. Predicting energy consumption of multiproduct pipeline using artificial neural networks. Energy 2014;66:791-798

[9] X. Yang. Design and Management of Oil Pipeline. China University of Petroleum Press, Dongying, Shandong; 2011.

[10] Wang, M. Yuan, Y. Yan, K. Yang, H. Zhang, Y. Liang. Optimal design of an oil pipeline with a large-slope section. Engineering Optimization 2018;51:1480-1494

[11] Swamee Prabhata. Design of a Submarine Oil Pipeline. Journal of Transportation Engineering 1993;119:159-170

[12] V. Vandeginste, K. Piessens. Pipeline design for a least-cost router application for $\mathrm{CO} 2$ transport in the CO2 sequestration cycle. International Journal of Greenhouse Gas Control 2008;2:571-581

[13] Xiaobo Luo, Meihong Wang, Eni Oko, Chima Okezue. Simulation-based techno-economic evaluation for optimal design of CO2 transport pipeline network. Applied Energy 2014;132:610-620

[14] N. Parker. Using Natural Gas Transmission Pipeline Costs to Estimate Hydrogen Pipeline Costs. Institute of Transportation Studies - University of California, Davis. 2004.

[15] Thomas J. Tarka. Estimating Carbon Dioxide Transport and Storage Costs. National Energy Technology Laboratory, Pittsburgh. 2010. 


\section{Author's Profile}

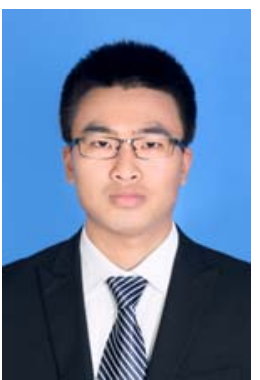

Wang Yuzhu, male, engineer, graduated from China University of Petroleum (East China) in 2015. He is currently working at the Oil and Gas Engineering Research Institute of Tarim Oilfield Branch, mainly engaged in current design management.

How to cite this paper: Yuzhu Wang, Xiaobo Mou, Yuanrui Zhang, Lande Cui, Wenlong Jia. "Study on Calculation Method of Economic Velocity of Gas Phase Ethane Conveying Pipeline", International Journal of Engineering and Manufacturing(IJEM), Vol.9, No.6, pp.1-13, 2019. DOI: 10.5815/ijem.2019.06.01 